J.Edu. Sci , Vol. (19) No.(3) 2007

\title{
The investigation of solutions for nonlinear system of differential equations with boundary conditions
}

\author{
R.N. Butris \& Azzam salahdin younis \\ Dept. of mathematics/ College of education/ Mosul University
}

Received

26/9/2006
Accepted

5/12/2006

\section{الخلاصة}

يظظمن البحث دراسة وجود وتقارب الحل لنظام من المعادلات التفاضلية اللاخطية ذات

شروط تخومية و ذلك باستخدام الطريقة التحليلية- العددية لبناء الحلول الدورية للمعادلات

التفاضلية الاعتيادية ـ Samoilenko A.M ـ كما تم من خلال هذا البحث تحسين الطريقة في

$$
\text { أعلاه و توسيعها. }
$$

\begin{abstract}
In this paper we investigate the existence and approximation for nonlinear system of differential equations with boundary conditions by using the numerical-analytic method for investigate of a periodic solutions which is given by Samoilenko A. M. and also these investigations lend us to the improving and extending the above method.
\end{abstract}




\section{Introduction:}

The numerical- analytic method has been used to study many boundary value problems $[1,2,3]$.

Samoilenko, A. M. and Ronto, N.I. used the numerical- analytic method for investigating of nonlinear system differential equation with boundary conditions:

$$
\begin{aligned}
& \frac{d x}{d t}=f(t, x) \\
& A x(0)+C x(T)=d
\end{aligned}
$$

Where the function $f(t, x)$ is continuous in $t, x$ on the domain:

$$
(t, x) \in[0, T] \times D
$$

$\mathrm{D}$ is closed domain subset of Euclidean spaces $R^{n}$

In this paper we using the numerical-analytic method of nonlinear system of differential equation with boundary conditions.

Also these investigations lend us to the improving and extending the above method.

Consider the following problem:

$$
\begin{aligned}
& \frac{d x}{d t}=f(t, x, y) \\
& A x(0)+C x(T)=d \\
& \frac{d y}{d t}=g(t, x, y) \\
& B y(0)+D y(T)=e
\end{aligned}
$$

Here $x \in D \subset R^{n}, y \in D_{1} \subset R^{m}, D$ and $D_{1}$ are closed domain subset of Euclidean spaces $R^{n}$ and $R^{m}$, respectively. Also $d=\left(d_{1}, d_{2}, \ldots ., d_{n}\right)$ and $e=\left(e_{1}, e_{2}, \ldots . e_{n}\right)$ are point in $R^{n}$. 
R.N. Butris \& Azzam salahdin younis

The vector functions

$$
\begin{aligned}
& f(t, x, y)=\left(f_{1}(t, x, y), \cdots \cdots, f_{n}(t, x, y)\right) \\
& g(t, x, y)=\left(g_{1}(t, x, y), \cdots \cdots \cdots, g_{n}(t, x, y)\right)
\end{aligned}
$$

are continuous on $\mathrm{t}, \mathrm{x}, \mathrm{y}$ on the domain

$$
(t, x, y) \in[0, T] \times D \times D_{1}
$$

and $A=\left(A_{i j}\right), C=\left(C_{i j}\right), E=\left(E_{i j}\right), D=\left(D_{i j}\right)$ are matrices $(\mathrm{n} \times \mathrm{n})$.

Let the functions $f(t, x, y), g(t, x, y)$ satisfies the following Lipschitz condition:

$\|f(t, x, y)\| \leq M,\|g(t, x, y)\| \leq N ;$

$\left\|f\left(t, x_{1}, y_{1}\right)-f\left(t, x_{2}, y_{2}\right)\right\| \leq \mathrm{K}_{1}\left\|\mathrm{x}_{1}-x_{2}\right\|+K_{2}\left\|\mathrm{y}_{1}-y_{2}\right\| ;$

$\left\|g\left(t, x_{1}, y_{1}\right)-g\left(t, x_{2}, y_{2}\right)\right\| \leq L_{1}\left\|x_{1}-x_{2}\right\|+L_{2}\left\|\mathrm{y}_{1}-y_{2}\right\|$

and $t \in[0, T], x, x_{1}, x_{2} \in D, y, y_{1}, y_{2} \in D_{1}$

and $K_{1}, K_{2}, L_{1}, L_{2}$ constants.

We define the non-empty sets as follows:

$$
\begin{aligned}
& D_{\beta}=D-\left(M \frac{T}{2}+B_{1}\right) ; \\
& D_{1 \beta}=D_{1}-\left(N \frac{T}{2}+B_{2}\right) ; \\
& B_{1}=\left\|C^{-1} d-\left(C^{-1} A+E\right) x_{0}\right\| \\
& B_{2}=\left\|D^{-1} e-\left(D^{-1} B+E\right) y_{0}\right\|
\end{aligned}
$$

Furthermore, we suppose that the largest equivalence $q$ of the following matrix. 
$Q(t)=\left(\begin{array}{lr}K_{1} \frac{\mathrm{T}}{2} & \mathrm{~K}_{2} \frac{\mathrm{T}}{2} \\ \mathrm{~L}_{1} \frac{\mathrm{T}}{2} & \mathrm{~L}_{2} \frac{\mathrm{T}}{2}\end{array}\right)$

is less than one.i.e.

$q=\frac{T / 2\left(K_{1}+L_{2}\right)+T \sqrt{\left(1 / 4\left(K_{1}+L_{2}\right)^{2}-\left(K_{1} L_{2}-L_{1} K_{2}\right)\right)}}{2} \leq 1$

\section{LEMMA 1}

Let $\mathrm{f}(\mathrm{t})$ be a continuous vector function in the interval $0 \leq t \leq T$, then

$\left\|\int_{0}^{t}\left(f(s)-\frac{1}{T} \int_{0}^{T} f(s) d s\right) d s\right\| \leq M \alpha(t)$

where

$$
\alpha(t)=2 t\left(1-\frac{t}{T}\right) \quad, \quad M=\max _{t \in[0, T]}|f(t)|
$$

\section{PROOF:}

From the following disparate we get

$$
\begin{aligned}
\left\|\int_{0}^{t}\left(f(s)-\frac{1}{T} \int_{0}^{T} f(s) d s\right) d s\right\| & \leq\left(1-\frac{t}{T}\right) \int_{0}^{t}\|f(s)\| d s+\frac{t}{T} \int_{t}^{T}\|f(s)\| d s \\
& \leq M \alpha(t)
\end{aligned}
$$

\section{Approximate solution}

The investigation of approximate solution of the problem (A) will be introduced by the following theorem.

\section{THEOREM 1}

If the system (A) satisfies the inequalities (1.5), (1.6), (1.7) and it have solutions $x=x\left(t, x_{0}, y_{0}\right)$ and $y=y\left(t, x_{0}, y_{0}\right)$ existence at the point $\left(t, x_{0}, y_{0}\right)$

then the sequence of the functions defined by: 


\section{R.N. Butris \& Azzam salahdin younis}

$$
\begin{aligned}
& x_{m}\left(t, x_{0}, y_{0}\right)=x_{0}+\int_{0}^{t}\left(f \left(s, x_{m-1}\left(s, x_{0}, y_{0}\right), y_{m-1}\left(s, x_{0}, y_{0}\right)-\right.\right. \\
& -\frac{1}{\mathrm{~T}} \int_{0}^{T} f\left(s, x_{m-1}\left(s, x_{0}, y_{0}\right), y_{m-1}\left(s, x_{0}, y_{0}\right) d s\right) d s+C^{-1} d-\left(C^{-1} A+E\right) x_{0} \\
& x_{0}\left(t, x_{0}, y_{0}\right)=x_{0},
\end{aligned}
$$

$\mathrm{m}=1,2 \ldots$

$$
\begin{aligned}
& y_{m}\left(t, x_{0}, y_{0}\right)=y_{0}+\int_{0}^{t}\left(g \left(s, x_{m-1}\left(s, x_{0}, y_{0}\right), y_{m-1}\left(s, x_{0}, y_{0}\right)-\right.\right. \\
& -\frac{1}{\mathrm{~T}} \int_{0}^{T} g\left(s, x_{m-1}\left(s, x_{0}, y_{0}\right), y_{m-1}\left(s, x_{0}, y_{0}\right) d s\right) d s+D^{-1} e-\left(D^{-1} B+E\right) y_{0}
\end{aligned}
$$$$
y_{0}\left(t, x_{0}, y_{0}\right)=y_{0},
$$

$\mathrm{m}=1,2 \ldots$

converges uniformly in the domain:

$$
\left(t, x_{0}, y_{0}\right) \in[0, T] \times D_{\beta} \times D_{1 \beta}
$$

for $x_{0} \in D_{\beta}, \quad y_{0} \in D_{1 \beta}$

to the functions $x_{\infty}\left(t, x_{0}, y_{0}\right), y_{\infty}\left(t, x_{0}, y_{0}\right)$ which are satisfying the integral equations

$$
\begin{aligned}
& x\left(t, x_{0}, y_{0}\right)=x_{0}+\int_{0}^{t}\left(f \left(s, x\left(s, x_{0}, y_{0}\right), y\left(s, x_{0}, y_{0}\right)-\right.\right. \\
& -\frac{1}{\mathrm{~T}} \int_{0}^{T} f\left(s, x\left(s, x_{0}, y_{0}\right), y\left(s, x_{0}, y_{0}\right) d s\right) d s+C^{-1} d-\left(C^{-1} A+E\right) x_{0} \ldots \\
& y\left(t, x_{0}, y_{0}\right)=y_{0}+\int_{0}^{t}\left(g \left(s, x\left(s, x_{0}, y_{0}\right), y\left(s, x_{0}, y_{0}\right)-\right.\right. \\
& -\frac{1}{\mathrm{~T}} \int_{0}^{T} g\left(s, x\left(s, x_{0}, y_{0}\right), y\left(s, x_{0}, y_{0}\right) d s\right) d s+D^{-1} e-\left(D^{-1} B+E\right) y_{0} \ldots(2 .
\end{aligned}
$$

its unique solution (1.3), (1.5) and satisfies the inequalities

$$
\left\|x_{\infty}\left(t, x_{0}, y_{0}\right)-x_{0}\right\| \leq M \frac{T}{2}+B_{1}
$$


$\left\|y_{\infty}\left(t, x_{0}, y_{0}\right)-y_{0}\right\| \leq N \frac{T}{2}+B_{2}$

\section{PROOF:}

Setting $\mathrm{m}=1$ and using lemma 1 and the sequence of the functions (2.12) we get

$$
\begin{gathered}
\left\|x_{1}\left(t, x_{0}, y_{0}\right)-x_{0}\right\| \leq\left(1-\frac{t}{T}\right) \int_{0}^{t}\left\|f\left(s, x_{0}, y_{0}\right)\right\| d s+\frac{t}{T} \int_{t}^{T}\left\|f\left(s, x_{0}, y_{0}\right)\right\| d s+ \\
+\left\|C^{-1} d-\left(C^{-1} A+E\right) x_{0}\right\| \\
\left\|x_{1}\left(t, x_{0} y_{0}\right)-x_{0}\right\| \leq M \alpha(t)+B_{1} \leq M \frac{T}{2}+B_{1}
\end{gathered}
$$

By lemma 1and the sequence of the functions (2.13) when $\mathrm{m}=1$ we get

$$
\begin{aligned}
& \left\|y_{1}\left(t, x_{0}, y_{0}\right)-y_{0}\right\| \leq\left(1-\frac{t}{T}\right) \int_{0}^{t}\left\|g\left(s, x_{0}, y_{0}\right)\right\| d s+\frac{t}{T} \int_{t}^{T}\left\|g\left(s, x_{0}, y_{0}\right)\right\| d s+ \\
& +\left\|D^{-1} e-\left(D^{-1} B+E\right) y_{0}\right\|, \\
& \left\|y_{1}\left(t, x_{0}, y_{0}\right)-y_{0}\right\| \leq N \alpha(t)+B_{2} \leq N \frac{T}{2}+B_{2} \\
& x_{1}\left(t, x_{0} y_{0}\right) \in D \text { for all } y_{0} \in D_{\beta 1}, x_{0} \in D_{\beta}, t \in[0, T]
\end{aligned}
$$

by induction we have:

$$
\begin{gathered}
\left\|x_{m}\left(t, x_{0}, y_{0}\right)-x_{0}\right\| \leq\left(1-\frac{t}{T}\right) \int_{0}^{t}\left\|f\left(s, x_{0}, y_{0}\right)\right\| d s+\frac{t}{T} \int_{t}^{T}\left\|f\left(s, x_{0}, y_{0}\right)\right\| d s+ \\
+\left\|C^{-1} d-\left(C^{-1} A+E\right) x_{0}\right\| \\
\left\|x_{m}\left(t, x_{0} y_{0}\right)-x_{0}\right\| \leq M \alpha(t)+B_{1} \\
\left\|y_{m}\left(t, x_{0}, y_{0}\right)-y_{0}\right\| \leq\left(1-\frac{t}{T}\right) \int_{0}^{t}\left\|g\left(s, x_{0}, y_{0}\right)\right\| d s+\frac{t}{T} \int_{t}^{T}\left\|g\left(s, x_{0}, y_{0}\right)\right\| d s+ \\
+\left\|D^{-1} e-\left(D^{-1} B+E\right) y_{0}\right\|
\end{gathered}
$$


$\left\|y_{m}\left(t, x_{0}, y_{0}\right)-y_{0}\right\| \leq N \alpha(t)+B_{2}$

$x_{m}\left(t, x_{0} y_{0}\right) \in D$ for all $y_{0} \in D_{\beta 1}, x_{0} \in D_{\beta}$

and $y_{m}\left(t, x_{0} y_{0}\right) \in D$ for all $y_{0} \in D_{\beta 1}, x_{0} \in D_{\beta}$

Now we prove the sequence of functions $\left\{x_{m}\left(t, x_{0}, y_{0}\right)\right\}_{m=0}^{\infty}$, $\left\{y_{m}\left(t, x_{0}, y_{0}\right)\right\}_{m=0}^{\infty}$ converges uniformly in the domain (1.7)

$$
\begin{gathered}
\left\|x_{m+1}\left(t, x_{0}, y_{0}\right)-x_{m}\left(t, x_{0}, y_{0}\right)\right\| \leq \alpha(t)\left[K_{1}\left\|x_{m}\left(s, x_{0}, y_{0}\right)-x_{m-1}\left(s, x_{0}, y_{0}\right)\right\|+\right. \\
\left.+K_{2}\left\|y_{m}\left(s, x_{0}, y_{0}\right)-y_{m-1}\left(s, x_{0}, y_{0}\right)\right\|\right]
\end{gathered}
$$

$$
\begin{aligned}
& \left\|y_{m+1}\left(t, x_{0}, y_{0}\right)-y_{m}\left(t, x_{0}, y_{0}\right)\right\| \leq \alpha(t)\left[L_{1}\left\|x_{m}\left(s, x_{0}, y_{0}\right)-x_{m-1}\left(s, x_{0}, y_{0}\right)\right\|+\right. \\
& \left.\quad+L_{2}\left\|y_{m}\left(s, x_{0}, y_{0}\right)-y_{m-1}\left(s, x_{0}, y_{0}\right)\right\|\right]
\end{aligned}
$$

Re write the inequalities (2.24), (2.23) in vector form as

$$
V_{m+1}(t) \leq Q(t) V_{m}(t)
$$

where

$$
\begin{aligned}
& V_{m+1}(t)=\left(\begin{array}{l}
\left\|x_{m+1}\left(t, x_{0}, y_{0}\right)-x_{m}\left(t, x_{0}, y_{0}\right)\right\| \\
\left\|y_{m+1}\left(t, x_{0}, y_{0}\right)-y_{m}\left(t, x_{0}, y_{0}\right)\right\|
\end{array}\right) ; \\
& Q(t)=\left(\begin{array}{lr}
K_{1} \frac{\mathrm{T}}{2} & \mathrm{~K}_{2} \frac{\mathrm{T}}{2} \\
\mathrm{~L}_{1} \frac{\mathrm{T}}{2} & \mathrm{~L}_{2} \frac{\mathrm{T}}{2}
\end{array}\right)
\end{aligned}
$$

and

$$
V_{m}(t)=\left(\begin{array}{l}
\left\|x_{m}\left(t, x_{0}, y_{0}\right)-x_{m-1}\left(t, x_{0}, y_{0}\right)\right\| \\
\left\|y_{m}\left(t, x_{0}, y_{0}\right)-y_{m-1}\left(t, x_{0}, y_{0}\right)\right\|
\end{array}\right) .
$$

it follows from inequality (2.25) that

$$
V_{m+1} \leq Q_{0} V_{m}
$$

where 


$$
Q_{0}=\max _{t \in[0, T]}|Q(t)|
$$

by iterating inequality (2.29) gives

$$
V_{m+1} \leq Q_{0}^{m} V_{1}
$$

which leads to the estimation

$$
\sum_{i=1}^{m} V_{i} \leq \sum_{i=1}^{m} Q_{0}^{i-1} V_{1}
$$

since the matrix $Q_{0}$ has eigenvalues (1.11).

Then the series (2.32) is uniformly convergent, i.e.

$$
\lim _{m \rightarrow \infty} \sum_{i=1}^{m} Q_{0}^{i-1} V_{1}=\sum_{i=1}^{\infty} Q_{0}^{i-1} V_{1}=\left(E-Q_{0}\right)^{-1} V_{1}
$$

The limiting relation (2.33) signifies a uniformly convergent of the sequence $\left[x_{m}\left(t, x_{0}, y_{0}\right), y_{m}\left(t, x_{0}, y_{0}\right)\right]$ in the domain (2.14)

Let:

$$
\left.\begin{array}{l}
\lim _{m \rightarrow \infty} x_{m}\left(t, x_{0}, y_{0}\right)=x_{\infty}\left(t, x_{0}, y_{0}\right) \\
\lim _{m \rightarrow \infty} y_{m}\left(t, x_{0}, y_{0}\right)=y_{\infty}\left(t, x_{0}, y_{0}\right)
\end{array}\right\}
$$

by inequality (2.31), the estimation

$$
\left(\begin{array}{l}
\left\|x_{\infty}\left(t, x_{0}, y_{0}\right)-x_{m}\left(t, x_{0}, y_{0}\right)\right\| \\
\left\|y_{\infty}\left(t, x_{0}, y_{0}\right)-y_{m}\left(t, x_{0}, y_{0}\right)\right\|
\end{array}\right) \leq Q_{0}^{m}\left(E-Q_{0}\right)^{-1} V_{1}
$$

is true for $m=1,2,3, \ldots$

Thus $x_{\infty}\left(t, x_{0}, y_{0}\right), y_{\infty}\left(t, x_{0}, y_{0}\right)$ are solutions of the integral equations (2.16), (2.15). 


\section{R.N. Butris \& Azzam salahdin younis}

Finally, we have to show that $x\left(t, x_{0}, y_{0}\right), y\left(t, x_{0}, y_{0}\right)$ are unique solution of (A)on country we suppose that there is at least two different solutions $\hat{y}\left(t, x_{0}, y_{0}\right), \hat{x}\left(t, x_{0}, y_{0}\right)$ of $(\mathrm{A})$.

From (1.15),(1.16) the following inequalities hold:

$$
\begin{aligned}
& \left\|x\left(t, x_{0}, y_{0}\right)-\hat{x}\left(t, x_{0}, y_{0}\right)\right\| \leq \alpha(t)\left[K_{1}\left\|x\left(s, x_{0}, y_{0}\right)-\hat{x}\left(s, x_{0}, y_{0}\right)\right\|+\right. \\
& \left.\quad+K_{2}\left\|y\left(s, x_{0}, y_{0}\right)-\hat{y}\left(s, x_{0}, y_{0}\right)\right\|\right] \\
& \left\|y\left(t, x_{0}, y_{0}\right)-\hat{y}\left(t, x_{0}, y_{0}\right)\right\| \leq \alpha(t)\left[L_{1}\left\|x\left(s, x_{0}, y_{0}\right)-\hat{x}\left(s, x_{0}, y_{0}\right)\right\|+\right. \\
& \left.+L_{2}\left\|y\left(s, x_{0}, y_{0}\right)-\hat{y}\left(s, x_{0}, y_{0}\right)\right\|\right]
\end{aligned}
$$

thus:

$$
\left(\begin{array}{l}
\left\|x\left(t, x_{0}, y_{0}\right)-\hat{x}\left(t, x_{0}, y_{0}\right)\right\| \\
\left\|y\left(t, x_{0}, y_{0}\right)-\hat{y}\left(t, x_{0}, y_{0}\right)\right\|
\end{array}\right) \leq Q_{0}\left(\begin{array}{l}
\left\|x\left(t, x_{0}, y_{0}\right)-\hat{x}\left(t, x_{0}, y_{0}\right)\right\| \\
\left\|y\left(t, x_{0}, y_{0}\right)-\hat{y}\left(t, x_{0}, y_{0}\right)\right\|
\end{array}\right)
$$

by iterating which we should find that

$$
\left(\begin{array}{l}
\left\|x\left(t, x_{0}, y_{0}\right)-\hat{x}\left(t, x_{0}, y_{0}\right)\right\| \\
\left\|y\left(t, x_{0}, y_{0}\right)-\hat{y}\left(t, x_{0}, y_{0}\right)\right\|
\end{array}\right) \leq Q_{0}^{m}\left(\begin{array}{l}
\left\|x\left(t, x_{0}, y_{0}\right)-\hat{x}\left(t, x_{0}, y_{0}\right)\right\| \\
\left\|y\left(t, x_{0}, y_{0}\right)-\hat{y}\left(t, x_{0}, y_{0}\right)\right\|
\end{array}\right)
$$

but $Q_{0} \rightarrow 0$ as $m \rightarrow \infty$ hence proceeding in the last inequality to the limit we obtain that $y\left(t, x_{0}, y_{0}\right)=\hat{y}\left(t, x_{0}, y_{0}\right), x\left(t, x_{0}, y_{0}\right)=\hat{x}\left(t, x_{0}, y_{0}\right)$ which proves the solution is unique and this completes the proof of theorem 1

\section{Existence of solution}

The problem of existence solution of $(\mathrm{A})$ is uniquely connected with the existence of zeros of the functions, $\Delta\left(0, x_{0}, y_{0}\right), \Delta^{*}\left(0, x_{0}, y_{0}\right)$ which has the form.

$\Delta\left(0, x_{0}, y_{0}\right)=\frac{1}{T}\left[\left(\mathrm{C}^{-1} \mathrm{~A}+\mathrm{E}\right) x_{0}-C^{-1} d\right]+\frac{1}{T} \int_{0}^{T} f\left(t, x_{\infty}\left(t, x_{0}, y_{0}\right), y_{\infty}\left(t, x_{0}, y_{0}\right)(3.40)\right.$ 
and

$\Delta^{*}\left(0, x_{0}, y_{0}\right)=\frac{1}{T}\left[\left(\mathrm{D}^{-1} \mathrm{~B}+\mathrm{E}\right) y_{0}-D^{-1} e\right]+\frac{1}{T} \int_{0}^{T} g\left(t, x_{\infty}\left(t, x_{0}, y_{0}\right), y_{\infty}\left(t, x_{0}, y_{0}\right)(3.41)\right.$

Since this functions are approximately determined from the sequence of functions:

$\Delta_{m}\left(0, x_{0}, y_{0}\right)=\frac{1}{T}\left[\left(\mathrm{C}^{-1} \mathrm{~A}+\mathrm{E}\right) x_{0}-C^{-1} d\right]+\frac{1}{T} \int_{0}^{T} f\left(t, x_{m}\left(t, x_{0}, y_{0}\right), y_{m}\left(t, x_{0}, y_{0}\right)(3.42)\right.$ and

$\Delta_{m}^{*}\left(0, x_{0}, y_{0}\right)=\frac{1}{T}\left[\left(\mathrm{D}^{-1} \mathrm{~B}+\mathrm{E}\right) y_{0}-D^{-1} e\right]+\frac{1}{T} \int_{0}^{T} g\left(t, x_{m}\left(t, x_{0}, y_{0}\right), y_{m}\left(t, x_{0}, y_{0}\right)\right.$

for $\mathrm{m}=0,1,2,3 \ldots$

\section{THEOREM 2}

Let all assumptions and conditions of theorem 1 were given then the following inequality

$\left(\begin{array}{l}\left\|\Delta\left(0, x_{0}, y_{0}\right)-\Delta_{m}\left(0, x_{0}, y_{0}\right)\right\| \\ \left\|\Delta^{*}\left(0, x_{0}, y_{0}\right)-\Delta_{m}^{*}\left(0, x_{0}, y_{0}\right)\right\|\end{array}\right) \leq \frac{2}{T} Q_{0}^{m+1}\left(E-Q_{0}\right)^{-1} V_{1}$,

Satisfies for $m \geq 0, y_{0} \in D_{\beta 1}, x_{0} \in D_{\beta}$.

\section{PROOF:}

By (3.40), (3.42) we get

$$
\begin{gathered}
\left\|\Delta\left(0, x_{0}, y_{0}\right)-\Delta_{m}\left(0, x_{0}, y_{0}\right)\right\| \leq \frac{1}{T} \int_{0}^{T} K_{1}\left\|x_{\infty}\left(t, x_{0}, y_{0}\right)-x_{m}\left(t, x_{0}, y_{0}\right)\right\|+ \\
+K_{2}\left\|y_{\infty}\left(t, x_{0}, y_{0}\right)-y_{m}\left(t, x_{0}, y_{0}\right)\right\| d t
\end{gathered}
$$

and by (3.41), (3.43) we get 


$$
\begin{aligned}
\| \Delta^{*}\left(0, x_{0}, y_{0}\right) & -\Delta^{*}{ }_{m}\left(0, x_{0}, y_{0}\right)\left\|\leq \frac{1}{T} \int_{0}^{T} L_{1}\right\| x_{\infty}\left(t, x_{0}, y_{0}\right)-x_{m}\left(t, x_{0}, y_{0}\right) \|+ \\
& +L_{2}\left\|y_{\infty}\left(t, x_{0}, y_{0}\right)-y_{m}\left(t, x_{0}, y_{0}\right)\right\| d t
\end{aligned}
$$

Re write the inequalities (3.45),(3.46)in vector forms as

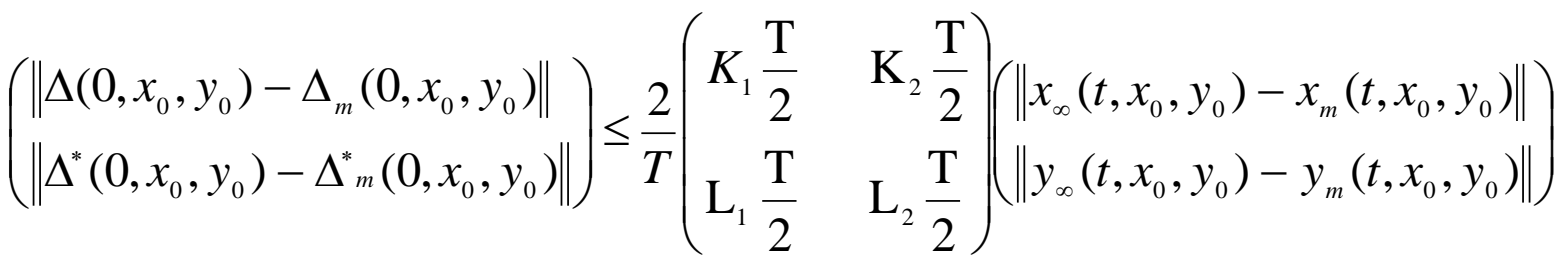

$$
\begin{aligned}
& \leq \frac{2}{T} Q_{0}^{m+1}\left(E-Q_{0}\right)^{-1} V_{1}
\end{aligned}
$$

Now we prove the following theorem taking into account that the inequality (3.44) will be satisfied for all $m \geq 0$.

\section{THEOREM 3}

Suppose that for $m \geq 0$ the sequence of functions $\Delta_{m}\left(0, x_{0}, y_{0}\right)$ and $\Delta_{m}^{*}\left(0, x_{0}, y_{0}\right)$ which are defined in (3.42), (3.43) satisfies the inequalities.

$$
\left(\begin{array}{l}
\min _{a+h \leq x_{0} \leq b-h} \Delta_{m}\left(0, x_{0}, y_{0}\right) \\
\min _{c+h^{*} \leq y_{0} \leq d-h^{*}} \Delta_{m}^{*}\left(0, x_{0}, y_{0}\right)
\end{array}\right) \leq-\sigma_{m}
$$

and

$$
\left(\begin{array}{c}
\max _{a+h \leq x_{0} \leq b-h} \Delta_{m}\left(0, x_{0}, y_{0}\right) \\
\max _{c+h^{*} \leq y_{0} \leq d-h^{*}} \Delta_{m}^{*}\left(0, x_{0}, y_{0}\right)
\end{array}\right) \geq \sigma_{m}
$$

when

$$
\sigma_{m}=\frac{2}{T} Q_{0}^{m+1}\left(E-Q_{0}\right)^{-1} V_{1}, V_{1}=\left(\begin{array}{c}
M \frac{T}{2}+B_{1} \\
N \frac{T}{2}+B_{2}
\end{array}\right) h=M \frac{T}{2}+B_{1}, h^{*}=N \frac{T}{2}+B_{2}
$$


Then the boundary problem (A) has solutions $x=x\left(t, x_{0}, y_{0}\right)$ $y=y\left(t, x_{0}, y_{0}\right)$ such that $a+h \leq x_{0} \leq b-h$ and $c+h^{*} \leq y_{0} \leq b-h^{*}$.

\section{PROOF:}

Let $x_{1}, x_{2}$ be any point in the interval $[a+h, b-h]$ such that

$$
\left.\begin{array}{l}
\Delta_{m}\left(0, x_{1}, y_{1}\right)=\min _{a+h x_{0} \leq b-h} \Delta_{m}\left(0, x_{0}, y_{0}\right) \\
\Delta_{m}\left(0, x_{2}, y_{2}\right)=\max _{a+h \leq x_{0} \leq b-h} \Delta_{m}\left(0, x_{0}, y_{0}\right)
\end{array}\right\}
$$

and let $y_{1}, y_{2}$ be any point in the interval $\left[c+h^{*}, d-h^{*}\right]$ such that

$$
\left.\begin{array}{l}
\Delta^{*}\left(0, x_{1}, y_{1}\right)=\min _{c+h^{*} \leq x_{0} \leq d-h^{*}} \Delta_{m}^{*}\left(0, x_{0}, y_{0}\right) \\
\Delta^{*}\left(0, x_{2}, y_{2}\right)=\max _{c+h^{*} \leq x_{0} \leq d-h^{*}} \Delta_{m}^{*}\left(0, x_{0}, y_{0}\right)
\end{array}\right\}
$$

by using the inqualities (3.47),(3.48),(3.49) we have:

$$
\begin{aligned}
& \left(\begin{array}{l}
\Delta\left(0, x_{1}, y_{1}\right) \\
\Delta^{*}\left(0, x_{1}, y_{1}\right)
\end{array}\right)=\left(\begin{array}{l}
\Delta_{m}\left(0, x_{1}, y_{1}\right)+\left[\Delta\left(0, x_{1}, y_{1}\right)-\Delta_{m}\left(0, x_{1}, y_{1}\right)\right] \\
\Delta^{*}{ }_{m}\left(0, x_{1}, y_{1}\right)+\left[\Delta^{*}\left(0, x_{1}, y_{1}\right)-\Delta^{*}{ }_{m}\left(0, x_{1}, y_{1}\right)\right]
\end{array}\right) \leq \overline{0} \\
& \left(\begin{array}{l}
\Delta\left(0, x_{2}, y_{2}\right) \\
\Delta^{*}\left(0, x_{2}, y_{2}\right)
\end{array}\right)=\left(\begin{array}{l}
\Delta_{m}\left(0, x_{2}, y_{2}\right)+\left[\Delta\left(0, x_{2}, y_{2}\right)-\Delta_{m}\left(0, x_{2}, y_{2}\right)\right] \\
\Delta^{*}\left(0, x_{2}, y_{2}\right)+\left[\Delta^{*}\left(0, x_{2}, y_{2}\right)-\Delta^{*}{ }_{m}\left(0, x_{2}, y_{2}\right)\right]
\end{array}\right) \geq \overline{0}
\end{aligned}
$$

From the continuity of (3.40), (3.41) and from (3.52), (3.53) there exists two point $x_{\infty}=x_{0}, x_{\infty} \in\left\lfloor x_{1}, x_{2}\right\rfloor$ and $y_{\infty}=y_{0}, y_{\infty} \in\left\lfloor y_{1}, y_{2}\right\rfloor$ such that: $\Delta\left(0, x_{\infty}, y_{\infty}\right)=0$ and $\Delta^{*}\left(0, x_{\infty}, y_{\infty}\right)=0$

thus $\quad x=x\left(t, x_{0}, y_{0}\right)$ is a solution for $x_{0} \in[a+h, b-h]$ and $y=y\left(t, x_{0}, y_{0}\right)$ is a solution for $y_{0} \in\left[c+h^{*}, d-h^{*}\right]$ of (A).

\section{REMARK 1}

When $R^{n}=R^{1}$ i.e. $x_{0}$ and $y_{0}$ are a scalar, theorem 2 can be strengthens by giving up the requirement that the singular point should be isolated. (For this remark see [3]) 
R.N. Butris \& Azzam salahdin younis

\section{THEOREM 3}

If the functions $\Delta\left(0, x_{0}, y_{0}\right)$ and $\Delta^{*}\left(0, x_{0}, y_{0}\right)$ are defined by

$$
\begin{gathered}
\Delta: D_{\beta} \rightarrow R^{n} \\
\Delta\left(0, x_{0}, y_{0}\right)=\frac{1}{T}\left[\left(\mathrm{C}^{-1} \mathrm{~A}+\mathrm{E}\right) x_{0}-C^{-1} d\right]+\frac{1}{T} \int_{0}^{T} f\left(t, x_{\infty}\left(t, x_{0}, y_{0}\right), y_{\infty}\left(t, x_{0}, y_{0}\right)(3.54)\right.
\end{gathered}
$$

and if

$$
\Delta^{*}: D_{\beta 1} \rightarrow R^{n}
$$

$\Delta^{*}\left(0, x_{0}, y_{0}\right)=\frac{1}{T}\left[\left(\mathrm{D}^{-1} \mathrm{~B}+\mathrm{E}\right) y_{0}-D^{-1} e\right]+\frac{1}{T} \int_{0}^{T} g\left(t, x_{\infty}\left(t, x_{0}, y_{0}\right), y_{\infty}\left(t, x_{0}, y_{0}\right)(3.55)\right.$

Where the functions $x_{\infty}\left(t, x_{0}, y_{0}\right)$ and $y_{\infty}\left(t, x_{0}, y_{0}\right)$ are limit of functions (2.12), (2.13) then the inequalities

$$
\begin{aligned}
& \left\|\Delta\left(0, x_{0}, y_{0}\right)\right\| \leq M+\frac{B_{1}}{T} \\
& \begin{array}{c}
\left\|\Delta\left(0, x_{0}^{1}, y_{0}^{1}\right)-\Delta\left(0, x_{0}^{2}, y_{0}^{2}\right)\right\| \leq K_{1}\left(1-E_{2}-E_{3} E_{6}\left(1-E_{4}\right)^{-1}\right)^{-1} . \\
.\left[E_{1}\left\|x_{0}^{1}-x_{0}^{2}\right\|+E_{5} E_{3}\left(1-E_{4}\right)^{-1}\left\|y_{0}^{1}-y_{0}^{2}\right\|\right]+ \\
+K_{2}\left(1-E_{4}-E_{3} E_{6}\left(1-E_{2}\right)^{-1}\right)^{-1}\left[E_{5}\left\|y_{0}^{1}-y_{0}^{2}\right\|+E_{6} E_{1}\left(1-E_{2}\right)^{-1}\left\|x_{0}^{1}-x_{0}^{2}\right\|\right]+ \\
+B_{1}\left\|x_{0}^{1}-x_{0}^{2}\right\|
\end{array}
\end{aligned}
$$

and

$$
\left\|\Delta^{*}\left(0, x_{0}, y_{0}\right)\right\| \leq N+\frac{B_{2}}{T}
$$




$$
\begin{gathered}
\left\|\Delta^{*}\left(0, x_{0}^{1}, y_{0}^{1}\right)-\Delta^{*}\left(0, x_{0}^{2}, y_{0}^{2}\right)\right\| \leq L_{1}\left(1-E_{2}-E_{3} E_{6}\left(1-E_{4}\right)^{-1}\right)^{-1} \cdot \\
\cdot\left[E_{1}\left\|x_{0}^{1}-x_{0}^{2}\right\|+E_{5} E_{3}\left(1-E_{4}\right)^{-1}\left\|y_{0}^{1}-y_{0}^{2}\right\|\right]+ \\
+L_{2}\left(1-E_{4}-E_{3} E_{6}\left(1-E_{2}\right)^{-1}\right)^{-1}\left[E_{5}\left\|y_{0}^{1}-y_{0}^{2}\right\|+E_{6} E_{1}\left(1-E_{2}\right)^{-1}\left\|x_{0}^{1}-x_{0}^{2}\right\|\right]+ \\
+B_{2}\left\|y_{0}^{1}-y_{0}^{2}\right\|
\end{gathered}
$$

for $y_{0}, y_{0}^{1}, y_{0}^{2} \in D_{\beta 1}, x_{0}, x_{0}^{1}, x_{0}^{2} \in D_{\beta}$.

\section{PROOF:}

From $x_{\infty}\left(t, x_{0}, y_{0}\right)$ and $y_{\infty}\left(t, x_{0}, y_{0}\right)$ then the functions $\Delta\left(0, x_{0}, y_{0}\right)$ and $\Delta^{*}\left(0, x_{0}, y_{0}\right)$ are continuous and bounded by $M+\frac{B_{1}}{T}$ and $N+\frac{B_{2}}{T}$ for $y_{0} \in D_{\beta 1}, x_{0} \in D_{\beta}$ we get

$$
\begin{aligned}
& \left\|\Delta\left(0, x_{0}^{1}, y_{0}^{1}\right)-\Delta\left(0, x_{0}^{2}, y_{0}^{2}\right)\right\| \leq \frac{1}{\mathrm{~T}} \int_{0}^{T} \| f\left(t, x_{\infty}\left(t, x_{0}^{1}, y_{0}^{1}\right), y_{\infty}\left(t, x_{0}^{1}, y_{0}^{1}\right)-\right. \\
& -f\left(t, x_{\infty}\left(t, x_{0}^{2}, y_{0}^{2}\right), y_{\infty}\left(t, x_{0}^{2}, y_{0}^{2}\right)\left\|d t+\frac{1}{T}\right\|\left(\mathrm{C}^{-1} \mathrm{~A}+\mathrm{E}\right) x_{0}^{1}-\left(\mathrm{C}^{-1} \mathrm{~A}+\mathrm{E}\right) x_{0}^{2} \| \ldots\right. \\
& \left\|\Delta\left(0, x_{0}^{1}, y_{0}^{1}\right)-\Delta\left(0, x_{0}^{2}, y_{0}^{2}\right)\right\| \leq K_{1}\left\|x_{\infty}\left(t, x_{0}^{1}, y_{0}^{1}\right)-x_{\infty}\left(t, x_{0}^{2}, y_{0}^{2}\right)\right\|+ \\
& +K_{2}\left\|y_{\infty}\left(t, x_{0}^{1}, y_{0}^{1}\right)-y_{\infty}\left(t, x_{0}^{2}, y_{0}^{2}\right)\right\|+B_{1}\left\|x_{0}^{1}-x_{0}^{2}\right\| \\
& \left\|\Delta^{*}\left(0, x_{0}^{1}, y_{0}^{1}\right)-\Delta^{*}\left(0, x_{0}^{2}, y_{0}^{2}\right)\right\| \leq \frac{1}{\mathrm{~T}} \int_{0}^{T} \| g\left(t, x_{\infty}\left(t, x_{0}^{1}, y_{0}^{1}\right), y_{\infty}\left(t, x_{0}^{1}, y_{0}^{1}\right)-\right. \\
& -g\left(t, x_{\infty}\left(t, x_{0}^{2}, y_{0}^{2}\right), y_{\infty}\left(t, x_{0}^{2}, y_{0}^{2}\right)\left\|d t+\frac{1}{T}\right\|\left(\mathrm{D}^{-1} \mathrm{~B}+\mathrm{E}\right) y_{0}^{1}-\left(\mathrm{D}^{-1} \mathrm{~B}+\mathrm{E}\right) y_{0}^{2} \| . .\right. \\
& \left\|\Delta^{*}\left(0, x_{0}^{1}, y_{0}^{1}\right)-\Delta^{*}\left(0, x_{0}^{2}, y_{0}^{2}\right)\right\| \leq L_{1}\left\|x_{\infty}\left(t, x_{0}^{1}, y_{0}^{1}\right)-x_{\infty}\left(t, x_{0}^{2}, y_{0}^{2}\right)\right\|+ \\
& +L_{2}\left\|y_{\infty}\left(t, x_{0}^{1}, y_{0}^{1}\right)-y_{\infty}\left(t, x_{0}^{2}, y_{0}^{2}\right)\right\|+B_{2}\left\|y_{0}^{1}-y_{0}^{2}\right\|
\end{aligned}
$$

Then the functions $x_{\infty}\left(t, x_{0}^{1}, y_{0}^{1}\right), x_{\infty}\left(t, x_{0}^{2}, y_{0}^{2}\right), y_{\infty}\left(t, x_{0}^{1}, y_{0}^{1}\right), y_{\infty}\left(t, x_{0}^{2}, y_{0}^{2}\right)$ are solutions of integral equations: 
$x\left(t, x_{0}^{k}, y_{0}^{k}\right)=x_{0}^{k}+\int_{0}^{t}\left(f\left(s, x\left(s, x_{0}^{k}, y_{0}^{k}\right), y\left(s, x_{0}^{k}, y_{0}^{k}\right)-\right.\right.$

$-\frac{1}{\mathrm{~T}} \int_{0}^{T} f\left(s, x\left(s, x_{0}^{k}, y_{0}^{k}\right), y\left(s, x_{0}^{k}, y_{0}^{k}\right) d s\right) d s+C^{-1} d-\left(C^{-1} A+E\right) x_{0}^{k} \ldots(3.64)$

and

$$
\begin{aligned}
& y\left(t, x_{0}^{k}, y_{0}^{k}\right)=y_{0}^{k}+\int_{0}^{t}\left(g \left(s, x\left(s, x_{0}^{k}, y_{0}^{k}\right), y\left(s, x_{0}^{k}, y_{0}^{k}\right)-\right.\right. \\
& -\frac{1}{\mathrm{~T}} \int_{0}^{T} g\left(s, x\left(s, x_{0}^{k}, y_{0}^{k}\right), y\left(s, x_{0}^{k}, y_{0}^{k}\right) d s\right) d s+D^{-1} e-\left(D^{-1} B+E\right) y_{0}^{k} .
\end{aligned}
$$

By (3.64) and lemma 1 we get

$$
\begin{aligned}
& \left\|x_{\infty}\left(t, x_{0}^{1}, y_{0}^{1}\right)-x_{\infty}\left(t, x_{0}^{2}, y_{0}^{2}\right)\right\| \leq\left(1+B_{1} T\right)\left\|x_{0}^{1}-x_{0}^{2}\right\|+ \\
& +\frac{T}{2} K_{1}\left\|x_{\infty}\left(t, x_{0}^{1}, y_{0}^{1}\right)-x_{\infty}\left(t, x_{0}^{2}, y_{0}^{2}\right)\right\|+\frac{T}{2} K_{2}\left\|y_{\infty}\left(t, x_{0}^{1}, y_{0}^{1}\right)-y_{\infty}\left(t, x_{0}^{2}, y_{0}^{2}\right)\right\| \ldots(3.66) \\
& \left\|y_{\infty}\left(t, x_{0}^{1}, y_{0}^{1}\right)-y_{\infty}\left(t, x_{0}^{2}, y_{0}^{2}\right)\right\| \leq\left(1+B_{2} T\right)\left\|y_{0}^{1}-y_{0}^{2}\right\|+ \\
& +\frac{T}{2} L_{1}\left\|x_{\infty}\left(t, x_{0}^{1}, y_{0}^{1}\right)-x_{\infty}\left(t, x_{0}^{2}, y_{0}^{2}\right)\right\|+\frac{T}{2} L_{2}\left\|y_{\infty}\left(t, x_{0}^{1}, y_{0}^{1}\right)-y_{\infty}\left(t, x_{0}^{2}, y_{0}^{2}\right)\right\| \ldots \text { (3.67) }
\end{aligned}
$$

Let

$$
\begin{aligned}
& \begin{array}{l}
E_{1}=\left(1+T B_{1}\right), E_{2}=\frac{T}{2} K_{1}, E_{3}=\frac{T}{2} K_{2}, \text { then } \\
\begin{aligned}
\left\|x_{\infty}\left(t, x_{0}^{1}, y_{0}^{1}\right)-x_{\infty}\left(t, x_{0}^{2}, y_{0}^{2}\right)\right\| & \leq\left(1-E_{2}\right)^{-1}\left[E_{1}\left\|x_{0}^{1}-x_{0}^{2}\right\|\right. \\
& \left.+E_{3}\left\|y_{\infty}\left(t, x_{0}^{1}, y_{0}^{1}\right)-y_{\infty}\left(t, x_{0}^{2}, y_{0}^{2}\right)\right\|\right]
\end{aligned} \\
\left.\begin{array}{r}
\left\|x_{\infty}\left(t, x_{0}^{1}, y_{0}^{1}\right)-x_{\infty}\left(t, x_{0}^{2}, y_{0}^{2}\right)\right\| \\
\quad+\left(1-E_{2}\right)^{-1} E_{1}\left\|x_{0}^{1}-x_{0}^{2}\right\|
\end{array}\right)^{-1} E_{3}\left\|y_{\infty}\left(t, x_{0}^{1}, y_{0}^{1}\right)-y_{\infty}\left(t, x_{0}^{2}, y_{0}^{2}\right)\right\|, \\
E_{4}=\frac{T}{2} L_{2}, E_{5}=\left(1+T B_{2}\right), E_{6}=\frac{T}{2} L_{1}, \\
\left\|y_{\infty}\left(t, x_{0}^{1}, y_{0}^{1}\right)-y_{\infty}\left(t, x_{0}^{2}, y_{0}^{2}\right)\right\| \leq\left(1-E_{4}\right)^{-1} E_{5}\left\|y_{0}^{1}-y_{0}^{2}\right\| \\
+\left(1-E_{4}\right)^{-1} E_{6}\left\|x_{\infty}\left(t, x_{0}^{1}, y_{0}^{1}\right)-x_{\infty}\left(t, x_{0}^{2}, y_{0}^{2}\right)\right\|
\end{array}
\end{aligned}
$$

from (3.70) in (3.66) we get 


$$
\begin{aligned}
&\left\|x_{\infty}\left(t, x_{0}^{1}, y_{0}^{1}\right)-x_{\infty}\left(t, x_{0}^{2}, y_{0}^{2}\right)\right\| \leq E_{1}\left\|x_{0}^{1}-x_{0}^{2}\right\|+E_{2}\left\|x_{\infty}\left(t, x_{0}^{1}, y_{0}^{1}\right)-x_{\infty}\left(t, x_{0}^{2}, y_{0}^{2}\right)\right\| \\
&+E_{5} E_{3}\left(1-E_{4}\right)^{-1}\left\|y_{0}^{1}-y_{0}^{2}\right\|+ \\
&+E_{3}\left(1-E_{4}\right)^{-1} E_{6}\left\|x_{\infty}\left(t, x_{0}^{1}, y_{0}^{1}\right)-x_{\infty}\left(t, x_{0}^{2}, y_{0}^{2}\right)\right\| \\
& \leq\left(1-E_{2}-E_{3} E_{6}\left(1-E_{4}\right)^{-1}\right)^{-1} \cdot \\
& . {\left[E_{1}\left\|x_{0}^{1}-x_{0}^{2}\right\|+E_{5} E_{3}\left(1-E_{4}\right)^{-1}\left\|y_{0}^{1}-y_{0}^{2}\right\|\right] \ldots(3.71) }
\end{aligned}
$$

from (3.69) in (3.67) we get

$$
\begin{gathered}
\left\|y_{\infty}\left(t, x_{0}^{1}, y_{0}^{1}\right)-y_{\infty}\left(t, x_{0}^{2}, y_{0}^{2}\right)\right\| \leq E_{5}\left\|y_{0}^{1}-y_{0}^{2}\right\|+E_{6} E_{1}\left(1-E_{2}\right)^{-1}\left\|x_{0}^{1}-x_{0}^{2}\right\|+ \\
+E_{6} E_{3}\left(1-E_{2}\right)^{-1}\left\|y_{\infty}\left(t, x_{0}^{1}, y_{0}^{1}\right)-y_{\infty}\left(t, x_{0}^{2}, y_{0}^{2}\right)\right\|+ \\
+E_{4}\left\|y_{\infty}\left(t, x_{0}^{1}, y_{0}^{1}\right)-y_{\infty}\left(t, x_{0}^{2}, y_{0}^{2}\right)\right\| \\
\leq\left(1-E_{4}-E_{3} E_{6}\left(1-E_{2}\right)^{-1}\right)^{-1} \cdot \\
\cdot\left[E_{5}\left\|y_{0}^{1}-y_{0}^{2}\right\|+E_{6} E_{1}\left(1-E_{2}\right)^{-1}\left\|x_{0}^{1}-x_{0}^{2}\right\|\right] . .
\end{gathered}
$$

Substituting (3.71) and (3.72) in (3.61) and (3.63), respectively. We get (3.57) and (3.59).

\section{REMARK 2}

The theorem 3 ensure the stability solution of the system (A) when there is a slight change in the points $x_{0}, y_{0}$ accompanied with a noticeable change in the functions $\Delta\left(0, x_{0}, y_{0}\right)$ and $\Delta^{*}\left(0, x_{0}, y_{0}\right)$.

(For this remark see [2])

\section{REFERENCE:}

1. Butris, R. N. Existence of a solution for system of second-order differential equation with Boundary conditions, Iraq, Mosul, J. Educ and sci vol (18) (1994).

2. Martynuk, C. B. The investigation of solution for differential equations with boundary conditions. Ukrain. Kiev. Nauk. nonlinear problems in the theory of differential equations N0 2 (1991).

3. Samoilenko, A. M. and Ronto, N. I. A numerical -analytic methods for investigations solutions with Boundary conditions Ukrain, Kiev, (1985). 